\title{
Les retombées de l'accident de Tchernobyl en France : analyse critique des mesures effectuées à l'époque sur le territoire national et enseignements pour la gestion de crise
}

\author{
PH. RENAUD ${ }^{1}$, D. LOUVAT ${ }^{1}$
}

(Manuscrit reçu le 19 décembre 2002, accepté le 2 juin 2003)

RÉSUMÉ Dix sept ans après l'accident de Tchernobyl, toutes les données de mesure de la radioactivité dans l'environnement faites à l'époque par les différents services de l'État ont été collationnées et validées. Ces données étaient appropriées pour évaluer dans des délais satisfaisants, les conséquences des retombées de cet accident pour la grande majorité de la population française. En revanche, elles ne permettaient pas, du fait de l'absence de prise en compte de l'importance des précipitations, d'évaluer la contamination des produits alimentaires locaux dans les régions de l'Est de la France où les dépôts ont été très supérieurs aux moyennes. Ainsi, la capacité de mesure mobilisée à la suite de cet accident n'a pu être focalisée sur des productions sensibles telles que les laits de brebis et de chèvre en Corse dont la consommation a pu conduire à des doses à la thyroïde des enfants significativement supérieures aux moyennes estimées pour l'Est de la France. Aujourd'hui, l'expérience de l'IRSN permettrait de mieux optimiser l'utilisation des moyens de mesures et de mieux assimiler les données produites. Toutefois cette optimisation ne pourra être complète sans que soit conduite l'analyse préalable de la sensibilité radioécologique des différentes composantes géographiques du territoire français qui doit guider toute stratégie d'échantillonnage.

ABSTRACT The Chernobyl fallout in France, critical review measurement-results obtained at that time and lessons learned for crisis management.

Seventeen years after, all data of radioactivity measurements made in the environment at the time of Chernobyl accident by the various official French organisations have been listed and reassessed. These data were appropriate to evaluate within a short period of time, the radiological consequences of the accident fallout for a large part of the French population. However, because the effect of rain was not taken into consideration, these data did not enabled a correct evaluation of the contamination of foodstuff in some parts of the Eastern France where radioactive deposition were exceeding the regional mean value. For instance, despite the large analytical capability devoted to sensitive foodstuff measurements as milk, no focus was placed on the consumption of Corsica goat and sheep milk which could have lead to thyroid doses for children significantly higher that estimated mean values for

\footnotetext{
1 IRSN, Scrvice d'études radiócologiques dans les milicux naturels, bâtiment I53, CE Cadarache, B.P. 3, 131 I5 Saint-PaulLez-Durance Cedex, France.
} 
Eastern France. Nowadays, the experience gained by IRSN would permit to better optimise the measurement distribution and to better assimilate data produced. However, to achieve this optimisation, we still need to carry on the radioecological sensitivity analysis of the different geographical compartments of the French territory, analysis eventually guiding any sample strategy.

L'accident nucléaire de Tchernobyl représente le cas particulier d'une situation post-accidentelle à gérer sans connaître les détails de la phase accidentelle qui permettent d'en appréhender la gravité. La pertinence de la réponse à ce type de situation repose sur un réseau de mesures qui rend compte rapidement et de manière appropriée de l'existence d'une pollution, de son niveau et de son ampleur sur le territoire national.

Dix sept ans après l'accident, l'IRSN a dressé un bilan de l'impact environnemental et dosimétrique de l'accident de Tchernobyl en France ( $w w w . i r s n . f r$ ). Toutes les données de mesure de la radioactivité faites à l'époque par les différents services de l'État ont été collationnées et validées et des modèles de transfert de la radioactivité au sein des écosystèmes ont été développés. D'un point de vue méthodologique, il est intéressant de confronter ce travail, fruit du retour d'expérience, avec la connaissance qu'ont eue les services de l'État, du développement de la contamination radioactive de l'environnement français dans les jours et les semaines qui ont suivi le 26 avril 1986. Le résultat de cette confrontation permet de tirer des enseignements pour la gestion de nouvelles crises post-accidentelles, en particulier sur la qualité et la disponibilité de l'information.

\section{1. Évaluer la contamination atmosphérique et les doses associées}

La détection des aérosols radioactifs ayant survolé le territoire après l'accident a été rapide. Les mesures des activités $\beta$-total (mesure de l'activité de tous les radionucléides émetteurs bêta) dans l'air de l'environnement des centres du Commissariat à l'énergie atomique (CEA) ont été effectuées dans les 24 heures qui ont suivi leur détection (entre le 29 et 30 avril). Dès le $1^{\text {er }}$ mai, on avait ainsi connaissance d'une élévation anormale de l'activité de l'air les 29 et 30 avril. Quelques heures après, des mesures plus précises donnaient les activités de plusieurs radionucléides, notamment les isotopes de l'iode, ${ }^{131} \mathrm{I}$ et ${ }^{132} \mathrm{I}$ (fils du ${ }^{132} \mathrm{Te}$ ), et le césium-137 ( $\left.{ }^{137} \mathrm{Cs}\right)$. Ainsi, de moins de $1 \mu \mathrm{Bq} \mathrm{m}{ }^{-3}$ avant le 29 avril, l'activité en ${ }^{137} \mathrm{Cs}$ de l'air est montée à quelques dizaines, voire centaines de $\mu \mathrm{Bq} \mathrm{m} \mathrm{m}^{-3}$ les 29 et 30 avril à Orsay, Verdun, Marcoule. Cette activité a ensuite atteint plusieurs $\mathrm{Bq} \mathrm{m^{-3 }}$ d'air entre le $1^{\text {er }}$ et le 2 mai sur la plupart des stations de l'Est de la France (7 Bq $\mathrm{m}^{-3}$ à Verdun) (IPSN, 1986). L'iode-131, inexistant dans l'air avant le passage des masses atmosphériques contaminées, a dépassé le $1^{\mathrm{er}}$ mai 

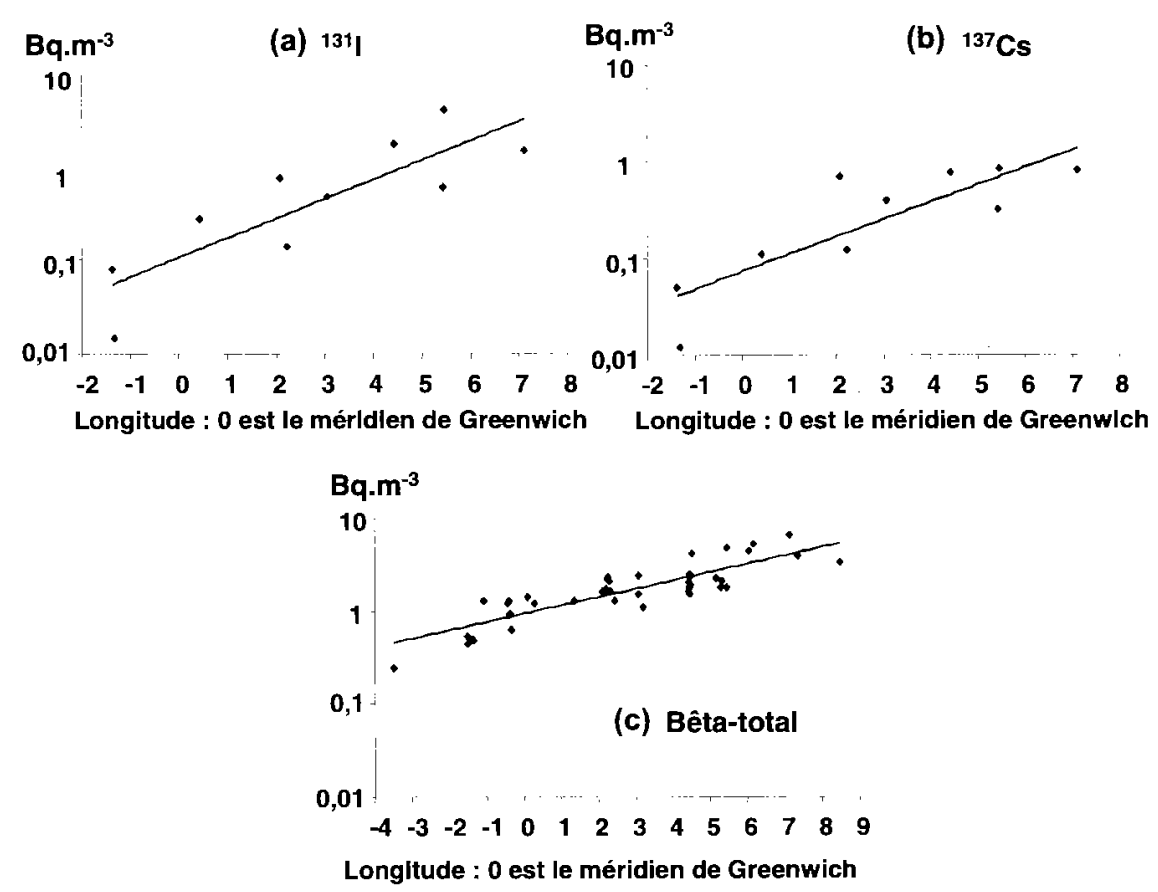

Figure 1 - Activités moyennes de l'air en ${ }^{131} I$ (a), ${ }^{137} \mathrm{Cs}$ (b) et $\beta$-total (c) entre le $1^{\text {er }}$ et le 3 mai 1986 en fonction de la longitude $(+)$ pour degrés est et (-) pour degrés ouest.

Mean air ${ }^{137} \mathrm{Cs},{ }^{131}$ I and beta-global activities in air between the Ist and the 3rd May 1986 with respect to longitude $(+)$ for east and (-) for west.

$10 \mathrm{~Bq} \mathrm{~m}^{-3}$ sur les stations de l'Est de la France. Parallèlement, les débits de dose enregistrés par le Service central de protection contre les rayonnements ionisants (SCPRI), exprimés alors en microrad par heure, ont augmenté de 30 à $50 \%$ dans l'Est du pays (SCPRI, 1986). La figure 1 présente les activités moyennes de l'air en ${ }^{137} \mathrm{Cs},{ }^{131}$ I et $\beta$-total entre le $1^{\text {er }}$ et le 3 mai 1986 en fonction de la longitude ; elle montre le gradient est-ouest de ces activités.

Par ailleurs, les services de protection radiologique (SPR) des installations nucléaires françaises ont participé à l'alerte et apporté un complément à l'information du réseau d'alerte du SCPRI. Ce réseau est aujourd'hui le réseau TÉLÉRAY de l'IRSN ( $w w w . i r s n . f r)$.

Les mesures d'aérosols atmosphériques effectuées principalement par le SCPRI et le CEA, permettaient la caractérisation des masses d'air contaminé très 
rapidement en fournissant une bonne indication des niveaux d'activité atteints. Ces mesures rendaient également compte de l'hétérogénéité de la contamination de l'air sur l'ensemble du territoire, et notamment de l'existence d'un gradient est-ouest. Enfin, elles permettaient d'évaluer dans des délais satisfaisants les doses reçues par la population lors du passage du nuage. Ces doses efficaces qui sont faibles, inférieures à $0,5 \mu \mathrm{Sv}$ pour l'irradiation externe et inférieures à $20 \mu \mathrm{Sv}$ pour l'inhalation (IPSN, 1986 ; Renaud et al., 1999a), ne nécessitaient pas à elles seules de dispositions particulières de protection de la population (mise à l'abri ou distribution d'iode stable). En effet, en 1986, les niveaux de doses, efficace et à la thyroïde, pour lesquels ces interventions étaient recommandées par la Commission internationale de protection radiologique (CIPR), étaient de 5 à $50 \mathrm{mSv}$ pour la dose efficace et de 50 à $500 \mathrm{mSv}$ pour la dose à la thyroïde (ICRP, 1984), la limite haute de la fourchette étant la valeur au-delà de laquelle des contre-mesures devaient être nécessairement appliquées et la limite basse de la fourchette la valeur en-deçà de laquelle la prise de contre-mesures n'est pas nécessaire. Entre ces deux niveaux de dose, des contre-mesures peuvent être prises en fonction de la situation et des conséquences secondaires qu'elles pouvaient induire.

\section{2. Évaluer le dépôt au sol}

Après l'évaluation des risques associés au passage même des masses d'air contaminé, c'est l'évaluation de la contamination de la chaîne alimentaire qui permet de juger de l'importance des conséquences des retombées pour la population et de prendre d'éventuelles mesures de protection telle que l'interdiction de consommation de produits frais. L'analyse de la contamination des chaînes alimentaires requiert soit une organisation d'échantillonnages et de mesures conséquentes, soit le cas échéant si celle-ci fait défaut une bonne connaissance des dépôts au sol. Cette connaissance, d'une part, constitue le point de départ de l'utilisation des modèles de transfert de la radioactivité dans l'environnement, d'autre part, guide la stratégie d'échantillonnage des produits agricoles pertinents pour une caractérisation plus fine de la situation.

Pour un accident de l'ampleur de celui de Tchernobyl, dont les retombées intéressent l'ensemble du territoire et peuvent présenter de grandes hétérogénéités, la connaissance de la diversité des dépôts par des mesures systématiques ne peut pas être exhaustive dans les premiers jours qui suivent ces dépôts. Pour rendre ainsi compte des hétérogénéités locales, notamment dans les zones de forts dépôts et faire porter l'effort de mesure sur ces zones, il est nécessaire tout d'abord de les localiser géographiquement. Dans le cas de Tchernobyl, cette localisation pouvait s'obtenir en tirant profit de la corrélation entre les hauteurs de précipitation et le degré de contamination. En effet, l'influence de la pluie sur l'importance des 
dépôts radioactifs est connue et assez bien quantifiée en radioécologie. C'est cette connaissance qui a conduit l'IPSN à effectuer des campagnes de mesures dans trois zones très pluvieuses la première semaine de mai 1986 au moment où les masses d'air contaminé ont survolé le pays : le Haut-Var (Maubert et al., 1988, 1997), le bassin de la Moselle (Delmas et Grauby, 1988), et le bassin du Tavignano en Corse (Ottavi et Charlent, 1988). Ces initiatives de l'IPSN, qui ont confirmé l'existence de zones de dépôts plus importants dans l'Est de la France, ont été tardives, le plus souvent à partir du mois de juin avec notamment très peu de mesures de ${ }^{131} \mathbf{I}$, et insuffisantes dans la mesure où elles n'ont pas permis de localiser toutes les zones concernées, ni leur extension.

L'obtention d'une carte précise des dépôts radioactifs et la localisation des zones les plus affectées passent donc par la connaissance et la prise en compte des hauteurs de précipitations. C'est cette information qui a fait le plus défaut dans la caractérisation de la situation en 1986. Il en a résulté la publication en mai 1986 d'estimations de dépôts fortement sous-évaluées puisqu'elles ne prenaient en compte que le dépôt par temps sec, qui dans l'Est de la France a souvent représenté moins de $10 \%$ du dépôt total.

Cette relation pluie-dépôt a permis d'aboutir à une carte des dépôts théoriques de ${ }^{137} \mathrm{Cs}$ et ${ }^{131} \mathrm{I}$ en 1986 (Fig. 2) (Renaud et al., 2003). Cette carte fait apparaître des dépôts supérieurs, voire très supérieurs aux estimations faites à l'époque, notamment sur toutes les zones de l'Est de la France ayant reçu des précipitations supérieures à $20 \mathrm{~mm}$. Si l'on excepte le premier bilan établi le 7 mai par le SCPRI, mentionnant des dépôts extrêmement faibles et inférieurs à $1000 \mathrm{~Bq} \mathrm{~m}^{-2}$, la première carte publiée par cet organisme dans son bulletin mensuel de juin 1986 (sorti quelques semaines plus tard), faisait état de dépôts moyens régionaux de ${ }^{137} \mathrm{Cs}$ allant de 1000 à $5400 \mathrm{~Bq} \mathrm{~m}{ }^{-2}$ dans l'Est de la France (SCPRI, 1986) alors qu'ils ont pu atteindre jusqu'à $40000 \mathrm{~Bq} \mathrm{~m}{ }^{-2}$.

Pour une évaluation correcte des dépôts radioactifs, il est donc nécessaire que les informations sur les précipitations atmosphériques venant du réseau de MétéoFrance soient exploitées dans les meilleurs délais par les experts gérant la situation post-accidentelle. À titre d'exemple, si en 1986, une partie de l'importante capacité de mesure mobilisée sur les départements de l'Ouest de la France où les retombées ont été très faibles, avait été reportée sur les zones très arrosées, nous disposerions de résultats de mesures permettant de mieux connaitre les niveaux de contamination atteints dans les productions locales qu'il est aujourd'hui très difficile de reconstituer précisément, surtout en ce qui concerne ${ }^{131}$ I. C'est le cas notamment en Corse où la production la plus sensible, celles des laits de chèvre et de brebis, n'a pas été échantillonnée et où les rares mesures sont celles effectuées 


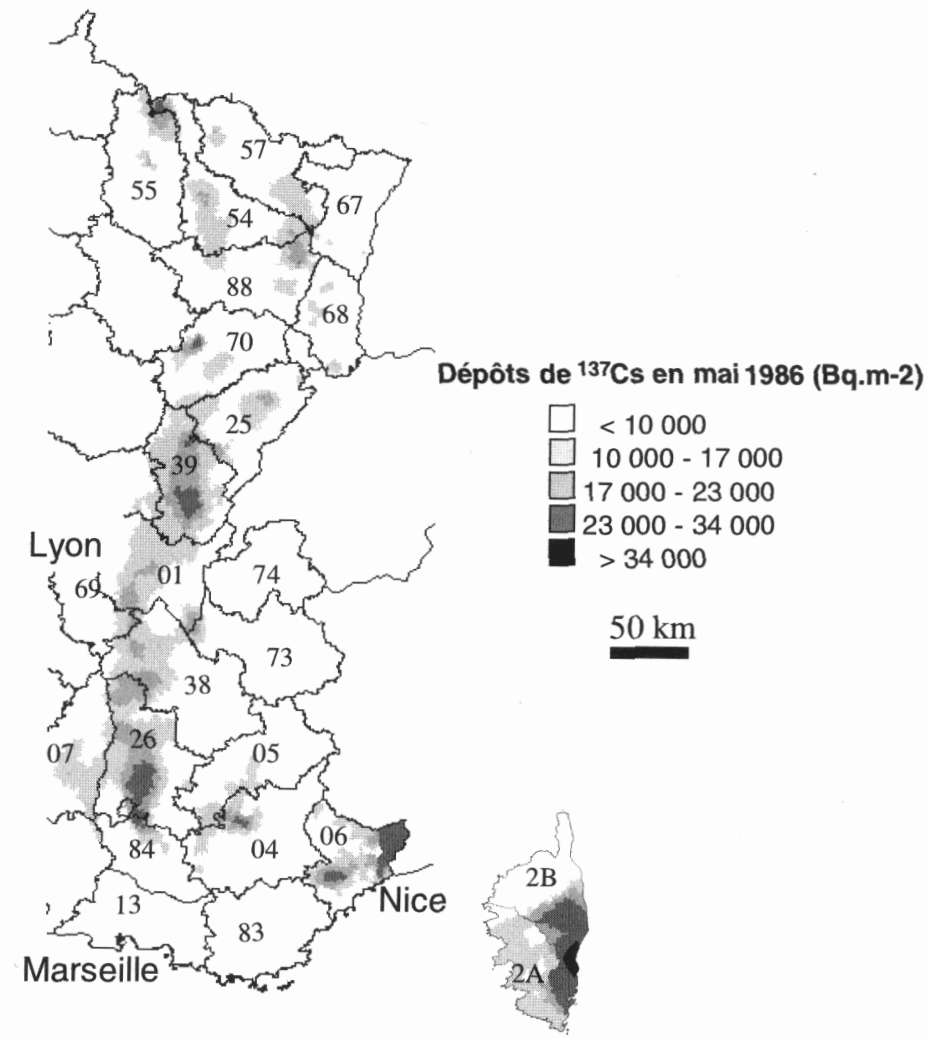

Figure 2-Carte des dépôts de ${ }^{137}$ Cs dans l'Est de la France (Renaud et al., 2003).

${ }^{137}$ Cs deposition map over the Eastern part of France (Renaud et al, 2003).

par l'IPSN et publiées par le SCPRI à la demande de particuliers qui ont fournis les échantillons (Renaud et al., 2002).

\section{3. Évaluer la contamination de la chaîne alimentaire}

\section{1. Évaluer la sensibilité radioécologique des zones les plus touchées}

Au-delà de la connaissance des dépôts, la gestion de la crise implique la caractérisation des zones affectées et notamment l'appréciation de leur sensibilité aux dépôts radioactifs (CEH, 2002). Cette sensibilité est liée aux facteurs qui vont influencer l'importance de l'atteinte de la population, de la chaîne alimentaire et 
de l'environnement au sens large : effectif et répartition de la population, degré de ruralité, niveaux d'autoconsommation et préparation des aliments, types de productions agricoles et leurs stades de développement au moment du dépôt, rémanence des dépôts dans les sols et des transferts dans la chaine alimentaire liée aux caractéristiques pédologiques, possibilités de prendre des dispositions destinées à limiter les conséquences radiologiques et économiques.

Dans le cas qui nous intéresse, les jours et semaines après les dépôts, la connaissance de la sensibilité des zones touchées permettrait d'optimiser la stratégie d'échantillonnage et de disposer des bons critères d'intervention postaccidentelle.

En 1986, compte tenu de la date de l'accident, la plupart des productions agricoles, fruitières et céréalières notamment, se trouvaient à un stade de développement très précoce et donc peu exposées aux dépôts radioactifs. Seuls deux groupes de produits étaient susceptibles de voir leur activité augmenter de manière significative : le lait et les produits laitiers, car les troupeaux étaient déjà à l'extérieur et consommaient des fourrages directement contaminés, et les légumes de saison cultivés en plein champ, notamment les légumes feuilles (salades, épinards, ...).

C'est sur ces deux produits que les mesures d'activités ont été les plus nombreuses. Les premiers prélèvements de lait ont été effectués assez tôt par le SCPRI, dès le 2 mai sur une dizaine de stations et dès le 5 mai sur les autres stations et dans les centres de collecte départementaux (SCPRI, 1986). Ce délai est compatible avec le temps moyen nécessaire pour que la majeure partie de l'activité ingérée par l'animal parvienne aux glandes mammaires ( 2 à 4 jours). Les prélèvements exceptionnels justifiés par la situation ont été nombreux, entre 3 et 10 prélèvements sur chaque station et dans chaque département, répartis sur le mois de mai. Ces prélèvements se sont prolongés durant les mois de juin et juillet. Ainsi plus de 500 analyses de lait ont été publiées dans les bulletins mensuels du SCPRI pour le seul mois de mai 1986, et près de 200 pour le mois de juin. Par ailleurs, près de 200 mesures significatives (au-dessus des limites de détection) de légumes feuilles (salades, épinards, poireaux) ont été effectuées au cours du mois de mai 1986 par le SCPRI pour le compte de la Direction générale de la consommation, de la concurrence et de la répression des fraudes (DGCCRF).

En 1986, l'analyse de la sensibilité radioécologique a été conduite sur la base de l'expérience du SCPRI mais sans le soutien d'un outil d'expertise approprié. Le développement d'un tel outil est actuellement à l'étude à I'IRSN. 


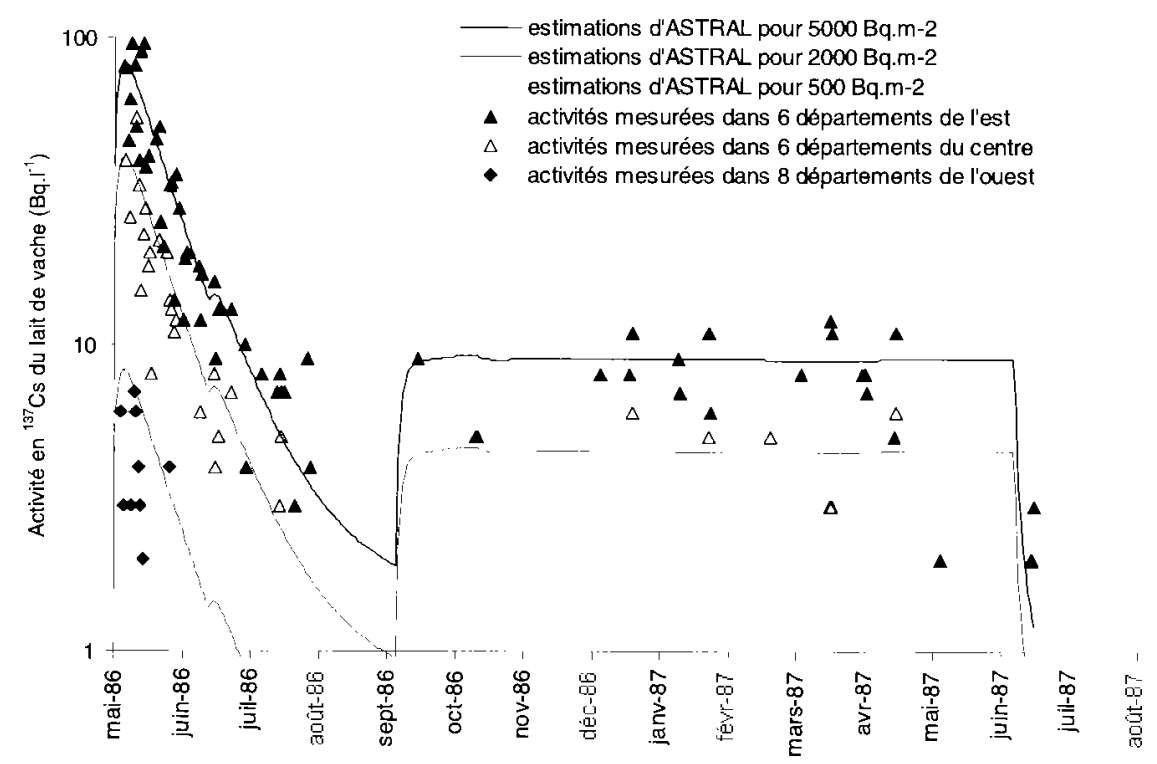

Figure 3 - Évolutions de l'activité moyenne départementale en ${ }^{137} \mathrm{Cs}$ du lait de vache dans 20 départements en 1986 et 1987.

${ }^{137}$ Cs cow milk mean activities from 20 districts in 1986 and 1987.

\section{2. Évaluer la contamination moyenne départementale}

À l'aide d'outils de calculs comme le modèle ASTRAL développé par l'IPSN (Renaud et al., 1999a), il est possible de vérifier la cohérence et donc la validité des mesures réalisées dans l'environnement et de les interpréter. En effet, à un dépôt radioactif correspond une évolution théorique des activités dans le lait et dans les légumes feuilles qui est assez bien connue. Il est ainsi possible de montrer que les mesures successives effectuées au cours des mois de mai et juin sur un même site sont cohérentes entre elles. De même, l'utilisation du modèle ASTRAL montre qu'il y a cohérence entre les activités mesurées dans le lait et celles mesurées dans les légumes au cours du temps. La figure 3 montre ainsi que l'activité du lait a rapidement répercuté les dépôts radioactifs et leur gradient est/ouest (Renaud et al., 1999b). Son évolution dans les mois qui ont suivi les dépôts s'accorde avec la diminution de l'activité de l'herbe consommée durant l'été, puis avec l'activité des fourrages consommés durant la période hivernale de stabulation mais récoltés au début de l'été. Dans l'Est de la France, les activités en ${ }^{137} \mathrm{Cs}$ du lait de vache publiées par le SCPRI et le Centre national d'études vétérinaires et alimentaires (CNEVA) sont inférieures à $220 \mathrm{~Bq}^{-1}$. 
L'étude des rapports d'activités isotopiques $\left({ }^{137} \mathrm{Cs}_{-}{ }^{134} \mathrm{Cs}^{-}{ }^{131} \mathrm{I}\right)$ témoigne également de la cohérence des données. En effet, dans toutes les composantes de l'environnement échantillonnées en mai et juin 1986, les rapports des activités ${ }^{137} \mathrm{Cs} /{ }^{134} \mathrm{Cs}$ mesurés étaient le plus souvent compris entre 1 et 4 pour une moyenne de 2 . Il en est de même des rapports ${ }^{131} \mathbf{I} /{ }^{137} \mathrm{C}$ s compris entre 2 et 18 pour l'air, 5 et 10 pour le lait avec une moyenne de 7,5 . On peut noter que cette variabilité du rapport ${ }^{131} \mathrm{I} /{ }^{137} \mathrm{Cs}$ n'est peut être pas le reflet d'une réalité mais plutôt un artefact résultant des différentes méthodes de mesures de l'iode.

L'ensemble de ces éléments permet de considérer que la plus grande partie des mesures de lait et de légumes feuilles réalisées après l'accident de Tchernobyl constituent une base fiable pour l'évaluation de la contamination moyenne de la chaîne alimentaire et des doses moyennes qui en ont résulté. Ceci n'exclut cependant pas que des valeurs plus importantes aient pu être atteintes localement. Les nombreuses autres mesures effectuées, dans les produits laitiers notamment, présentent un intérêt mojndre. Elles sont certes indicatives des niveaux atteints dans ces produits, mais sont difficiles à interpréter, à valider et à relier aux autres mesures. Elles résultent en effet de traitements agroalimentaires susceptibles de modifier considérablement les concentrations des radionucléides et s'accompagnent de délais de production difficiles à apprécier. Elles sont cependant globalement cohérentes avec les mesures de lait.

Certaines denrées comme les céréales, les viandes et les fruits, ont fait l'objet d'un nombre insuffisant de mesures pour permettre de reconstituer partout leur niveau de contamination moyen. Leurs activités ont pu être néanmoins calculées à partir de la carte des dépôts moyens départementaux sur les surfaces agricoles, carte déduite au moyen d'ASTRAL, des mesures de lait et de légumes feuilles. En effet, la mesure d'un échantillon de lait prélevé dans un grand centre de collecte départemental est représentative de l'activité moyenne d'une grande quantité du lait produite à cette échelle et surtout des dépôts moyens sur de très grandes surfaces de prairie. La carte ainsi obtenue (Fig. 4) est le reflet de la contamination moyenne du lait. Elle fait apparaître 4 zones sur lesquelles les activités mesurées dans le lait sont assez homogènes. Ceci est en accord avec la diminution d'est en ouest de l'activité des masses d'air (Fig. 1) et du lait (Fig. 3). Les rares mesures de céréales et de fruits disponibles ont permis ensuite de valider partiellement, les valeurs théoriques obtenues par le calcul sur la base de cette carte. Si elle est satisfaisante pour rendre compte des dépôts «efficaces » sur les prairies et les végétaux cultivés, et donc de la contamination moyenne de la chaîne alimentaire dans son ensemble, cette carte ne peut être considérée comme représentative des dépôts au sol dans les zones les plus arrosées durant la première semaine de mai 1986. Lors de sa sortie en 1997, elle était d'ailleurs accompagnée de commentaires sur la sous-estimation des dépôts au sol sur ces zones. Cette sous-estimation ne 


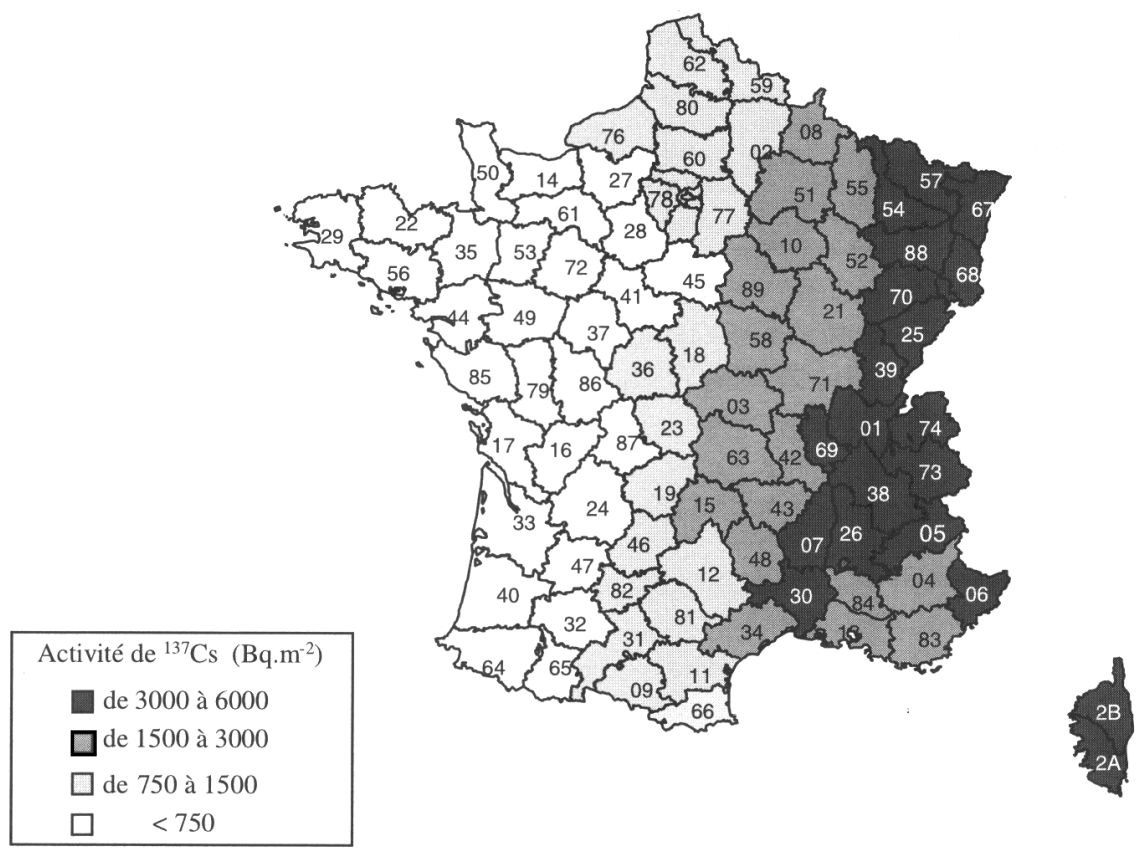

Figure 4-Carte des dépôts moyens départementaux de ${ }^{137} \mathrm{Cs}$ sur les surfaces agricoles, déduite des mesures de lait et de légumes feuilles effectuées en mai 1986.

${ }^{137}$ Cs mean depositions on agricultural surfaces, based on cow milk and leafy vegetables activities measured in May 1986.

pouvait être quantifiée à l'époque. Aujourd'hui, à la lumière des travaux ultérieurs de reconstitution des dépôts ( $c f$. paragraphe 2), il apparaît que pour certains départements comme le Jura ou la Drôme, les dépôts moyens ont pu être jusqu'à trois fois supérieurs à ceux figurant dans cette carte.

La contamination en ${ }^{131}$ I de la chaîne alimentaire peut être estimée de la même manière à partir des nombreuses mesures de ce radionucléide effectuées dans le lait et les légumes. La carte des dépôts moyens d ${ }^{131}$ I pour les surfaces agricoles ressemble à celle du ${ }^{137} \mathrm{Cs}$ avec des activités 5 à 10 fois supérieures. Les activités des autres radionucléides comme ${ }^{134} \mathrm{Cs}$ et ${ }^{106} \mathrm{Ru}$ peuvent être déduites des rapports d'activité isotopiques ponctuellement mesurés.

Une telle utilisation du modèle ASTRAL, qui n'existait pas en 1986, facilite le travail d'évaluation de la contamination moyenne de la chaîne alimentaire. Le niveau de précision qu'offre un outil comme ASTRAL est lié à la finesse de 
l'estimation des dépôts au sol et à la pertinence de la stratégie d'échantillonnage. En 1986, les zones où les dépôts ont été les plus forts n'ont donné lieu qu'à des mesures occasionnelles. Pourtant, très tôt, quelques mesures avaient témoigné d'activités très supérieures aux moyennes observées dans l'Est de la France : $2750 \mathrm{~Bq} \mathrm{~kg}^{-1} \mathrm{~d}^{131} \mathrm{I}$ dans une salade prélevée à Mandelieu (06) le 2 mai (Maubert et al., 1988), $2600 \mathrm{~Bq} \mathrm{~kg}^{-1}$ dans de la viande prélevée par le CNEVA dans la $2^{\mathrm{e}}$ semaine de mai, $4400 \mathrm{~Bq}{ }^{-1} \mathrm{~d}^{131}$ I le 12 mai dans du lait de chèvre corse (SCPRI, 1986).

\section{4. Évaluer les doses dues à l'ingestion de denrées contaminées}

Aux faibles doses reçues par la population lors du passage des masses d'air contaminées (paragraphe 1), il faut ajouter celles liées à l'ingestion et à l'irradiation par les dépôts.

En se fondant sur les mesures effectuées dans le lait et les légumes en 1986, la dose efficace moyenne engagée cette année là par ingestion de ces produits et de leurs dérivés est estimée, pour des comportements alimentaires moyens, à $150 \pm$ $50 \mu \mathrm{Sv}$ pour la population résidant dans l'Est de la France; voisine de celle déterminée en Suisse $(230 \mu \mathrm{Sv})$ (CFSR, 1987) c'est-à-dire près de $80 \%$ de la dose moyenne globale due à l'ingestion de l'ensemble des produits alimentaires évaluée au moyen du modèle ASTRAL : soit $190 \pm 80 \mu \mathrm{Sv}$. Cette estimation faite en 1997 est proche de celles faites en 1986 par l'IPSN pour les habitants de la région de Dijon et basées sur les mesures effectuées dans l'environnement du centre CEA de Valduc : $170 \mu \mathrm{Sv}$.

Le lait et les légumes, consommés entre mai et juillet 1986, contribuent par ailleurs à la majeure partie des doses à la thyroïde. Pour les enfants de l'Est de la France, la dose moyenne à la thyroïde est estimée suivant leur âge entre 2 et $10 \mathrm{mSv}$ (Vidal et Renaud, 2000). Ces doses sont également proches de celle évaluée en 1986 pour les enfants âgés de 10 ans habitant la région du centre CEA de Valduc : 6,6 mSv. En Suisse, ces mêmes doses ont été estimées à $11 \mathrm{mSv}$ pour les enfants âgés de 10 ans et $35 \mathrm{mSv}$ pour les enfants âgés de 2 ans.

En Corse, la région la plus affectée par l'accident, cette dose de $10 \mathrm{mSv}$ n'a pas dû être dépassée de manière importante pour une majorité des enfants. Des doses plus importantes ont cependant pu être reçues par des enfants ayant consommé à cette époque des produits frais provenant de zones où les dépôts ont été les plus forts comme la plaine orientale de l'île, ou encore par des enfants qui avaient un régime alimentaire particulier. Dans ce cas, les doses à la thyroïde ont pu dépasser $50 \mathrm{mSv}$ et atteindre $150 \mathrm{mSv}$ (Renaud et al., 2002). 
L'existence de ces doses plus élevées, dans les zones les plus affectées, ont été mentionnées par l'IPSN dès le mois de décembre 1986, ces doses à la thyroïde pouvant atteindre $90 \mathrm{mSv}$ pour des enfants corses (Lettre Dir-IPSN, 1986). Les rares mesures effectuées en 1986 dans les zones de forts dépôts restent encore aujourd'hui une base étroite pour estimer les doses maximales susceptibles d'avoir été reçues à l'époque.

De manière générale, les estimations de doses locales effectuées en 1986, notamment pour les personnes résidant dans les environs des centres du CEA, sont proches de celles faites depuis 1997. Pourtant, ce n'est qu'en 1996 que les facteurs de doses aux public et notamment aux enfants, paramètres clefs de l'estimation des doses, ont été publiés au Journal officiel des communautés européennes (JO-CE, 1996). En 1986, les estimations de doses ont été effectuées en utilisant des facteurs de doses issus d'un rapport scientifique allemand (Henrichs et al., 1985). Les travaux de la CIPR pour l'obtention de ces facteurs en fonction de l'âge et pour des formes physico-chimiques identiques à celles rencontrées dans l'environnement, n'ont commencé qu' après l'accident de Tchernobyl (Métivier, 2000). Les résultats de ces travaux ont conduit à plusieurs publications successives et recommandations de la CIPR avant d'être repris au Journal officiel des communautés européennes (Directive 96/29/Euratom du 13/05/1996). En ce qui concerne les coefficients de dose à la thyroïde, les écarts entre la publication allemande de 1985 et celle du JO-CE du 29 juin 1996 sont faibles, inférieures à $20 \%$. Dans le cas des doses efficaces, ceux de 1996 peuvent être jusqu'à 2 fois supérieurs à ceux utilisés en 1986.

\section{Conclusion}

Les organismes officiels, notamment le Service central de protection contre les rayonnements ionisants, ont effectué un grand nombre de mesures de radioactivité dans l'environnement français à la suite de l'accident de Tchernobyl.

Transmises et interprétées au fur et mesure de leur obtention, ces mesures étaient appropriées pour évaluer dans des délais satisfaisants, les conséquences des retombées de cet accident pour la grande majorité de la population française.

En revanche, ces mesures ne permettaient pas d'évaluer la contamination des produits locaux de la chaîne alimentaire, notamment en iode-131, dans les régions du pays où les dépôts ont été les plus importants, du fait de l'absence de prise en compte des précipitations qui ont conduit à des dépôts hétérogènes et localement très supérieurs aux moyennes. Les doses susceptibles d'avoir été reçues par des personnes ayant consommé ces productions locales ne peuvent donc être évaluées, encore aujourd'hui que de manière théorique. L'utilisation de mesures d'activités actuelles de ${ }^{137} \mathrm{Cs}$ dans les sols étant hasardeuse (Renaud et al., 2003), 
ces évaluations, qui reposent sur la reconstitution des dépôts sur la base des pluies enregistrées en mai 1986 et les rares mesures effectuées à cette époque, restent délicates et limitées. Ainsi, à partir de la carte des dépôts théoriques de ${ }^{137} \mathrm{Cs}$ et ${ }^{131}$ I en Corse, de quelques mesures de lait de et de légumes feuilles ainsi que d'une mesure de la charge thyrö̈dienne en ${ }^{131}$ I d'un enfant Corse effectuées en juillet 1986, une évaluation rétrospective de l'ordre de grandeur des doses à la thyroïde reçues par les enfants sur l'ensemble de l'île et des valeur maximales susceptibles d'avoir été atteinte a pu être effectuées. Si les doses moyennes à la thyroïde, estimées à $10 \mathrm{mSv}$ pour les enfants de l'Est de la France, n'ont pas été dépassées de manière importante par la majeure partie des enfants de Corse, des doses plus importantes ont pu être reçues par des enfants qui, à cette époque, ont consommé des produits frais provenant de zones où les dépôts ont été les plus forts comme la plaine orientale corse ou par des enfants qui avaient un régime alimentaire particulier. Dans ce cas, les doses à la thyroïde ont pu dépasser $50 \mathrm{mSv}$ et atteindre $150 \mathrm{mSv}$ (Renaud et al., 2002).

Aujourd'hui l'IRSN dispose d'outils supplémentaires qui permettent une interprétation rapide des données de terrain. Au travers de cette confrontation entre la situation actuelle et celle de 1986, il apparaît qu'il faut encore progresser dans le domaine de l'optimisation de l'utilisation des moyens de mesures et d'intervention par une analyse préalable de la vulnérabilité des différentes composantes géographiques du territoire français. Il s'agit d'étudier les facteurs environnementaux susceptibles d'augmenter ou de réduire les conséquences d'un rejet sur la population, la chaîne alimentaire et l'environnement de manière générale.

\section{RÉFÉRENCES}

CEH (2002) Radioecological Sensitivity. Final Report from the Center for Ecology and Hydrology. Ed Howard B.K. Oxford.

CFSR (1987) $29^{\mathrm{e}}$ rapport de la Commission fédérale de surveillance de la radioactivité : 1985-1986, Form 311.310 .

Delmas J., Grauby A. (1988) Impact et dynamique de la radioactivité provenant de l'accident de Tchernobyl dans trois bassins versants, IV Symposium international de radioécologie de Cadarache, 11-18 mars 1988 , tome 1, section D, 35-48.

Henrichs K., Elsasser U., Schotola C., Kaul A. (1985) Dosisfaktoren für inhalation oder ingestion von radionuklidverbindungen, ISH-Heft 80, Bundesgesundheitsamt Institut für Strahlen hygiene.

ICRP publication 40 (1984) Protection of the public in the cvent of Major Radiation Accidents principles for planning, Ann. ICRP 14(2).

IPSN (1986) L'accident de Tchernobyl, rapport IPSN n²/86, CEA-IPSN juin 1986.

JO-CE (1996) Directive 96/29/Euratom du conseil du 13 mai 1996 fixant les normes de base relatives à la protection sanitaire de la population et des travailleurs contre les dangers résultants des rayonnements ionisants, Journal officiel des communautés européennes L159. 
Lettre Dir-IPSN (1986) Lettre du Directeur de l'IPSN au Dr. Fauconnier, Réf. 1380/86/IPSN du $8 / 12 / 1986$

Maubert H., Roussel S., Lion R. (1988) Les dépôts consécutifs à l'accident de Tchernobyl dans le bassin du Var, IV Symposium international de radioécologie de Cadarache, 11-18 mars 1988, tome 1 , section D, pp. 24-33

Maubert H., Jourd'heuil L., Métivier J.M. (1997) Note sur les valeurs de radioactivité mesurées dans le Haut bassin du Var, réédition du rapport DPRE-97-04.

Métivier H. (2000) Les bases de la réglementation, Ann. Ass. Belg. Radioprot. 25(2), 99-113.

Ottavi L., Charlent O. (1988) Étude du bassin versant du Tavignano après les retombées de l'accident de Tchernobyl, NE Symposium de Cadarache, 11-18 mars 1988.

Renaud Ph., Stapel R., Maubert H., Bleher M., Wirth E. (1999a) Comparative study of the PARK and ASTRAL post-accidental decision support software, Health Phys. 76(5), 502-509.

Renaud Ph., Beaugelin K., Maubert H., Ledenvic Ph. (1999b) Les retombées en France de l'accident de Tchernobyl, conséquences radioécologiques et dosimétriques. Éditions de Physique, Les Ulis.

Renaud Ph., Robeau D., Vidal M., Pourcelot L. (2002) Les retombées de l'accident de Tchernobyl en Corse : contamination de la châne alimentaice et doses à la thyroïde associée, rapport IRSN 02-22.

Renaud Ph., Pourcelot L., Métivier J.M., Morello M. (2003) Mapping of ${ }^{137} \mathrm{Cs}$ deposition over eastern France 16 years after the Chernobyl accident, Sci. Tot. Environmt. 309, 257-264.

SCPRI (1986) Bulletins mensuels de résultats de mesures du Service Central de Protection contre les Rayonnements Ionisants, mai, juin et juillet 1986.

Vidal M., Renaud Ph. (2000) Estimation des doses moyennes à la thyroüde reçues par les enfants en France à la suite de l'accident de Tchernobyl et analyse de sensibilité, note technique SEGR/SAER/00-67. 